ORIGINAL ARTICLE

\title{
Partially hydrolysed guar gum supplemented comminuted chicken diet in persistent diarrhoea: a randomised controlled trial
}

\author{
N H Alam, R Meier, S A Sarker, P K Bardhan, H Schneider, N Gyr
}

Arch Dis Child 2005;90:195-199. doi: 10.1136/adc.2003.040089

See end of article for authors' affiliations authors affiliations

Correspondence to: Dr N H Alam, Clinical Sciences Division, International Centre for Diarrhoeal Disease Research, GPO Box 128 Dhaka 1000, Bangladesh; nhalam@icddrb.org

Accepted 26 April 2004

\begin{abstract}
Background: Partially hydrolysed guar gum (Benefiber) added to a diet is fermented in the colon, producing short chain fatty acids, which improve intestinal function, including colonic salt and water absorption.

Aims: To evaluate the effect of Benefiber supplemented comminuted chicken diet in the treatment of persistent diarrhoea.

Methods: One hundred and sixteen children (aged 5-24 months), presenting to Dhaka Hospital with a history of watery diarrhoea for more than 14 days (persistent diarrhoea), were randomised to receive either: (1) comminuted chicken diet with Benefiber (study diet); or (2) comminuted chicken diet without Benefiber (control diet). The study period was seven days.

Results: Of 116 children, 57 received the study diet and 59 received the control diet. Diarrhoea resolved in a greater number of children with the study than with the control diet (46/55 (84\%) v 36/58 (62\%); odds ratio $3.12,95 \% \mathrm{Cl} 1.19$ to 8.4$)$. Survival analysis for the duration of diarrhoea also showed a reduced duration of diarrhoea in children receiving the study diet. There was also a trend in daily stool reduction in children receiving the study diet, significant on days 4-7.

Conclusion: Results show that Benefiber supplemented comminuted chicken diet enhances recovery of children with persistent diarrhoea, indicating its therapeutic potential.
\end{abstract}

$\mathrm{P}$ ersistent diarrhoea, defined as a continuous diarrhoeal episode for 14 days or more, ${ }^{1}$ is a major clinical problem encountered by physicians in third world countries. Studies in several developing countries have shown that 3$20 \%$ of acute diarrhoeal episodes in children under 5 years of age become persistent. ${ }^{1}$ A wide spectrum of disturbances occurring in persistent diarrhoea has been noted, and it appears that the pathogenesis of this syndrome is multifactorial. Suggested mechanisms in the pathogenesis of persistent diarrhoea include: (a) persistence of acute diarrhoeal pathogens; (b) continued mucosal injury; (c) delayed mucosal repair; and (d) bacterial overgrowth. ${ }^{1}$ However, it seems that, whatever is the primary insult to the intestinal tract, small intestinal mucosal damage is a major feature of the pathophysiology. ${ }^{2}$ Depending on the severity of mucosal damage, changes occur in the digestive, secretory, absorptive, and reabsorptive capacities of the mucosa for macro and micronutrients. Considering these functional disturbances of the small intestine, modified diets have been used for the management of persistent diarrhoea in the Dhaka Hospital of the International Centre for Diarrhoeal Disease Research Bangladesh (ICDDR) and other centres. Colonic function in persistent diarrhoea has not been studied. The colon plays an important role for conserving water and electrolytes. ${ }^{3}$ Water movement across the colonic epithelium is not dependent on active transport, but is passive in response to solute absorption. Short chain fatty acids (SCFAs) are the predominant anions in the colonic lumen, and are major contributors to solute transport. ${ }^{4-6}$

SCFAs are microbial breakdown products of dietary fibres/ unabsorbed carbohydrates and are rapidly absorbed. Although antimicrobials are not routinely indicated in persistent diarrhoea except where there is severe malnutrition, indiscriminate use of broad spectrum antimicrobials in persistent diarrhoea and modified diets without polysaccharide/dietary fibres, in addition to diarrhoeal flow, means that the colonic concentration of SCFAs may be decreased. Thus reduction of luminal concentration of SCFAs might result in colonic dysfunction with impaired colonic water and electrolyte absorption leading to prolongation of diarrhoeal episodes. A soluble and fermentable fibre, such as partially hydrolysed guar gum (Benefiber, Taiyo Kagaku Co., Japan), if added to the diet in the treatment of persistent diarrhoea, might help to improve colonic function. This product has been shown to be efficacious in the treatment of acute diarrhoea in children. ${ }^{7}$ The present study was carried out to evaluate the efficacy of a Benefiber supplemented comminuted chicken diet in the management of persistent diarrhoea in children.

\section{PATIENTS AND METHODS}

The study was carried out at the Dhaka Hospital of ICDDR (Centre for Health and Population Research) from February 1999 to December 2000; the protocol was approved by the ICDDR's Research Review and Ethical Review Committees. Patients eligible for the study were male children between the age of 5 and 24 months, with a history of watery diarrhoea lasting for more than 14 days. Children excluded from the study were those with signs of associated diseases (for example, pneumonia, septicaemia, meningitis), gross electrolyte imbalance, altered mental status, and severe malnutrition. Informed consent was obtained from the parent(s) or legal guardian before enrolment of each patient in the study.

Children remained in the hospital until diarrhoea resolved. Initial evaluation included recording of detailed medical

Abbreviations: ICDDR, International Centre for Diarrhoeal Disease Research; ORS, oral rehydration solution; PHGG, partially hydrolysed guar gum; SCFA, short chain fatty acid 


\begin{tabular}{|lcc|}
\hline \multicolumn{2}{|l|}{ Table 1 Admission characteristics of the study children } & \\
\hline Variables & $\begin{array}{l}\text { Study diet } \\
\mathbf{n}=57\end{array}$ & $\begin{array}{c}\text { Control diet } \\
\mathbf{n}=59\end{array}$ \\
\hline Age (mth) & $9.0(2.9)$ & $8.4(3.0)$ \\
Duration of diarrhoea before admission (days) & $20.2(5.6)$ & $20.7(10.7)$ \\
Frequency of stool in previous 24 h before admission & $10.9(4.0)$ & $10.2(3.9)$ \\
Frequency of vomiting in previous 24 h before admission & $2.0(2.1)$ & $1.6(2.0)$ \\
Wt for age Z score & $-1.9(0.9)$ & $-2.0(1.0)$ \\
Breast fed (no.) & $54 / 57$ & $55 / 59$ \\
Dehydration status (no.), no sign:some & $35: 22 / 57$ & $34: 25 / 59$ \\
Stool pathogen (no.) & & \\
Shigella & $0 / 57$ & $2 / 59$ \\
Salmonella & $1 / 57$ & $1 / 59$ \\
Campylobacter iejuni & $8 / 57$ & $11 / 59$ \\
Vibrio cholerae & $0 / 57$ & $0 / 59$ \\
Aeromonas sobria & $4 / 57$ & $6 / 59$ \\
Aeromonas hydrophila & $3 / 57$ & $2 / 59$ \\
No bacterial pathogen identified & $41 / 57$ & $33 / 59$ \\
Rotavirus & $25 / 57$ & $15 / 59$ \\
\hline Values are mean (SD) or numbers, unless specified. & & \\
\hline
\end{tabular}

history and thorough physical examination, including anthropometry. Dehydration was assessed according to the WHO guideline. ${ }^{8}$ Laboratory investigations included stool microscopic examination for leucocytes, red blood cells, and parasites (including Giardia lambia, Entamoeba histolytica, and Cryptosporidium); stool was cultured for Shigella, Salmonella, Vibrio cholerae, Aeromonas, and Campylobacter jejuni by standard methods, and detection of rotavirus by enzyme linked immunosorbent assay (ELISA) as a part of routine laboratory tests for management of persistent diarrhoea patients.

\section{Sample size}

The sample size was estimated based on a recently conducted study (PK Bardhan et al, personal communication) at the ICDDR in which $65 \%$ of children receiving the comminuted chicken diet recovered within seven days. A sample size of 60 children per group was selected with the expectation of a $90 \%$ recovery within seven days with comminuted chicken diet supplemented with Benefiber, assuming a $20 \%$ withdrawal rate, at a $5 \%$ level of significance and $80 \%$ power.

\section{Treatment allocation/randomisation}

After enrolment, children were randomly assigned to one of the two dietary treatment groups: (1) Benefiber supplemented comminuted chicken diet (study diet), defined in the next section under "Diet"; or (2) comminuted chicken diet (control diet). A randomisation list was prepared for an intervention to control ratio $1: 1$ using a random number table with permuted blocks of 20 and compiled by a statistician not involved in the study. Serially numbered sealed identical envelopes were also prepared by the statistician containing the allotted diet. The envelopes were supplied to the dietician of the hospital who prepared the diets according to the list. The two diets were identical in appearance, smell, and taste. For analysis a code (group A or B) was provided to the investigators, without disclosing the patients' identities. After extensive analysis and preparation of tables, group identity was disclosed for preparation of the final report.

\section{Case management}

\section{Fluid treatment}

After admission, the children were rehydrated with the standard WHO oral rehydration solution (ORS) (10-12 ml/kg body weight per hour) during a period of 4-6 hours. Ongoing stool losses were replaced with the same ORS with a volume at least equal to the loss of watery stool until diarrhoea ceased. The prepared ORS was discarded after 12 hours if the whole amount $(0.5 \mathrm{l})$ was not consumed within this period.

\section{Diet}

Breast milk was allowed as desired. Supplementary feeding was given in the form of comminuted chicken diet (minced chicken, soy oil, glucose mixture, $60 \mathrm{kcal} / 100 \mathrm{~g}$ ). The study group received comminuted chicken diet with $20 \mathrm{~g} / \mathrm{l}$ of Benefiber, and the control group received comminuted chicken diet without Benefiber.

\section{Micronutrients}

All children received 10 multivitamin drops twice daily, zinc acetate $5 \mathrm{mg}$ three times daily, and folic acid $1.2 \mathrm{mg}$ daily orally for at least two weeks as standard hospital practice. In addition, children above 1 year of age and children 612 months of age were given 200000 IU and 100000 IU respectively of vitamin A on admission.

\section{Measurements}

The children were placed on a "cholera cot", and a paediatric urine collector was applied to collect urine separately. Stool weight, supplemented food (comminuted chicken diet), and body weight were measured with an electronic scale (Sartorius, Gottingen, Germany) with a precision of $1 \mathrm{~g}$. ORS, plain water, and urine were measured with a calibrated cylinder. All intake (ORS, plain water, and food) and output

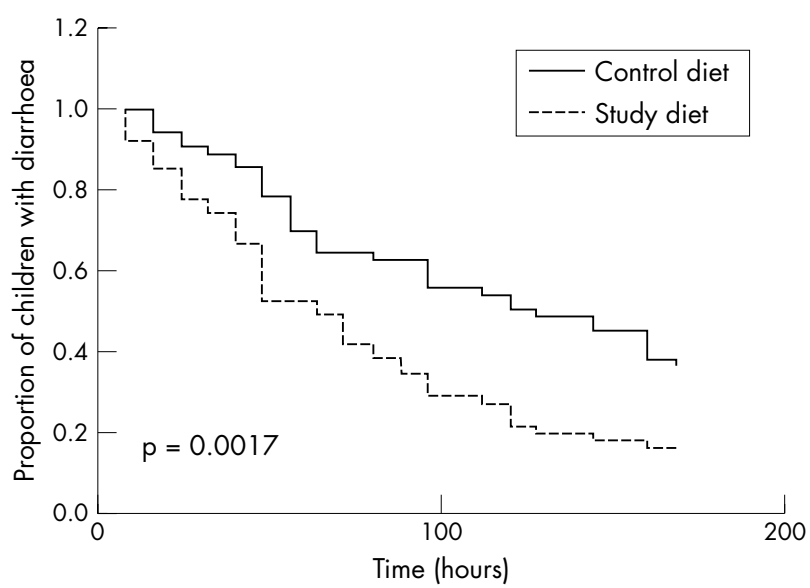

Figure 1 Survival plot for recovery from diarrhoea. 


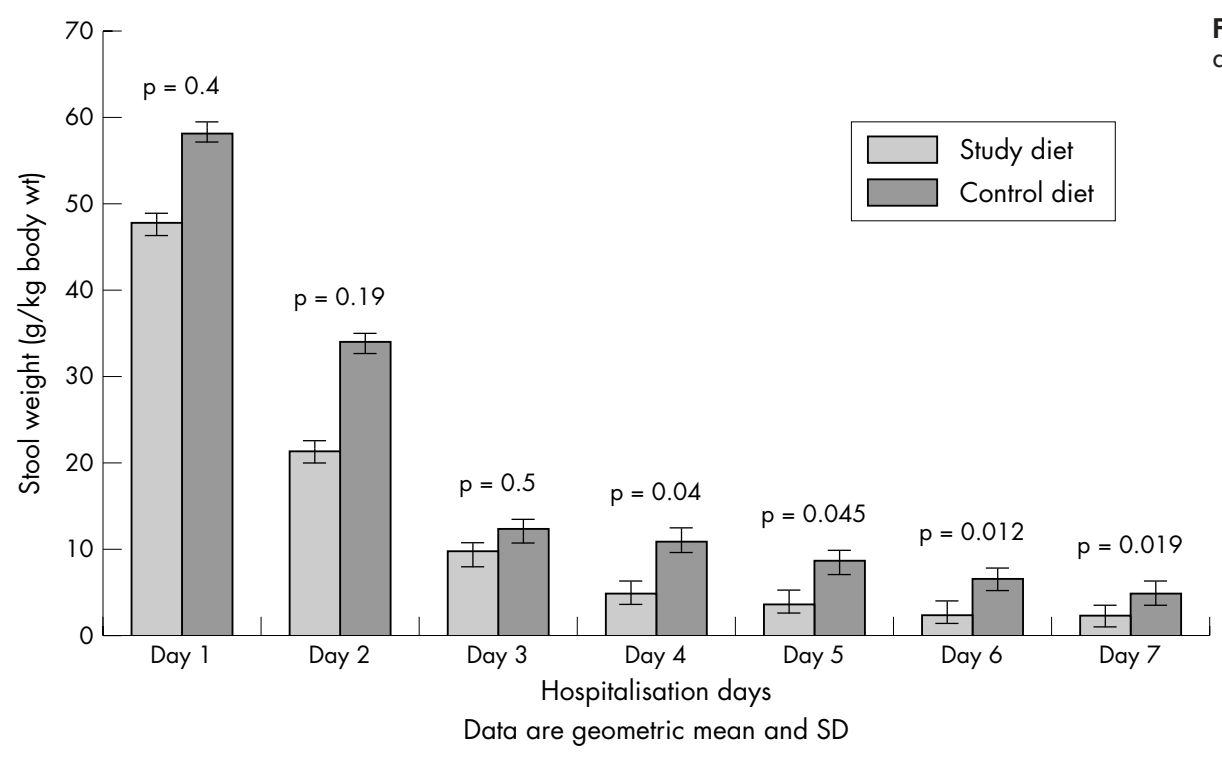

Figure 2 Stool weight daily up to 7 days.

(stool, urine, and vomit) measurements were summarised every eight hours. Body weight was also measured every eight hours until the children were discharged. Clinical evaluation was performed every morning and evening. Cessation of diarrhoea was defined as the passage of stool of normal consistency over a period of at least 48 hours. Therapeutic success was defined as the cessation of diarrhoea within seven days of inclusion in the study treatment. Duration of diarrhoea was calculated as the duration in hours from the time of randomisation to the last watery or loose stool within seven days. Children were withdrawn from the study if their parents or legal guardian withdrew consent, or if transferred to other units for treatment of severe underlying illness; data of such patients up to the point of withdrawal were included in the analysis. Data of the children who failed to recover within the study period (seven days) were also included in the analysis for a maximum of seven days; those were labelled as therapeutic failures and were treated in the hospital until recovery.

\section{Clinical outcome}

Main outcome measures were resolution of diarrhoea within seven days and daily stool output.

\section{Data analysis}

All data were analysed by means of a microcomputer using SPSS PC+ (version 10). Baseline characteristics of the study groups were compared using Student's $t$ test for continuous variables and the $\chi^{2}$ test for categorical variables. Outcome data of continuous variables were compared between groups using Student's $t$ test, and a non-parametric test was used when data were not normally distributed. Categorical variables were compared using the $\chi^{2}$ test. Also continuous data such as stool weight, urine output, vomit, ORS intake, and water intake were log transformed (because the distribution was not normal) before applying the Student's $t$ test. Kaplan-Meier survival curves were constructed to compare duration of diarrhoea; comparison was by the log rank test; a p value $<0.05$ was considered statistically significant. All analyses were performed on the "intent to treat" data set.

\section{RESULTS}

In total, 158 children were screened for the study, of whom 116 children were randomised; 57 received comminuted chicken diet with Benefiber (study diet) and 59 received comminuted chicken diet without Benefiber (control diet). Reasons for non-randomisation included failure to meet study inclusion criteria $(\mathrm{n}=34)$ and refusal of consent $(n=8)$. Three children were withdrawn from the study by the parents before completion of the study-two from the study diet and one from the control diet.

Baseline clinical characteristics such as age, body weight, duration of diarrhoea before admission, breast feeding status, and pathogens isolated were comparable between the groups (table 1). Although rotavirus detection in stool was more

Table 2 Clinical outcome of the study children

\begin{tabular}{|c|c|c|c|c|}
\hline Variables & $\begin{array}{l}\text { Study diet } \\
n=57\end{array}$ & $\begin{array}{l}\text { Control diet } \\
n=59\end{array}$ & $\begin{array}{l}\text { Difference between means } \\
(95 \% \mathrm{Cl})\end{array}$ & $\mathrm{p}$ value \\
\hline $\begin{array}{l}\text { No. }(\%) \text { children stopped diarrhoea } \\
\text { within } 7 \text { days }\end{array}$ & $46 / 55(84)$ & $36 / 58(62)$ & $3.12(1.19$ to 8.4$) \dagger$ & $0.02^{*}$ \\
\hline Total stool, $\mathrm{g} / \mathrm{kg} \ddagger$ & $525(177)$ & 615 (359) & $-90.00(-278$ to 92.26$)$ & 0.456 \\
\hline Total urine, $\mathrm{ml} / \mathrm{kg} \ddagger$ & $298(233)$ & $416(211)$ & $-118.51(-272.76$ to 35.72$)$ & 0.111 \\
\hline Total ORS, ml/kgł & 492 (195) & 638 (403) & $-146.69(-356.65$ to 63.27$)$ & 0.283 \\
\hline Total water, $\mathrm{ml} / \mathrm{kg} \ddagger$ & $330(144)$ & $353(172)$ & $-22.67(-142.26$ to 96.90$)$ & 0.716 \\
\hline Total calories, kcal/kg/day & $47.0(13.1)$ & $49.5(13.0)$ & $-2.51(-8.19$ to 3.16$)$ & 0.381 \\
\hline
\end{tabular}

Values are mean (SD) or number (\%), unless specified.

*Statistically significant.

tOdds ratio $(95 \% \mathrm{Cl})$.

¥Cumulative data until recovery of diarrhoea or maximum 7 days. 
frequent in the study group, the difference was not statistically significant. Tests for diarrhoeagenic $E$ coli were not performed.

A significantly greater number of children on the study diet had resolution of diarrhoea within seven days than children on the control diet: $46 / 55(84 \%) v 36 / 58$ (62\%) (odds ratio $3.12,95 \%$ CI 1.19 to $8.4, p=0.02$ ) (table 2 ). The survival analysis for the duration of diarrhoea also showed a similar trend towards reduced duration of diarrhoea in children receiving the study diet $(p=0.0017, \log$ rank test; fig 1$)$. There was also a trend for reduction in daily stool weight in children receiving the study diet; however, the differences were statistically significant only from days $4-7(\mathrm{p}=0.041$, $0.045,0.014,0.022$, respectively; fig 2). Cumulative stool weight $(\mathrm{g} / \mathrm{kg}$ body weight) from day 1 to day 7 , although reduced in the study group, was not significantly different (table 2 ). Total ORS intake (ml/kg body weight) and energy intake (kcal/kg body weight per day) from supplemented diets were not significantly different between the groups. Total urine output ( $\mathrm{ml} / \mathrm{kg}$ body weight) and water intake ( $\mathrm{ml} /$ $\mathrm{kg}$ body weight) were also similar in both groups (table 2 ).

\section{DISCUSSION}

The study shows that Benefiber, when added to comminuted chicken diet, enhances recovery of children suffering from persistent diarrhoea. Similar results were also reported when Benefiber supplemented, WHO recommended, glucose based oral rehydration solution was used in the treatment of acute diarrhoea in children compared with the treatment without Benefiber. ${ }^{7}$ In both studies, the subjects' age and nutritional status were similar. Although persistent diarrhoea is recognised as a separate entity, it is thought to be initiated by an intestinal infection; however, isolation of pathogen is less frequent in persistent diarrhoea. The impact of Benefiber was modest with regard to reduction of stool output. Whether increasing the dose of Benefiber ( $50 \mathrm{~g} / \mathrm{l}$ ) could be beneficial, is beyond the scope of this study; however, further studies with different doses might be useful in determining the optimal dose for maximising the effect of Benefiber in the management of persistent diarrhoea. Consistent with our hypothesis, a recent study by Ramakrishna and colleagues ${ }^{9}$ has shown that amylase resistant starch, derived from maize, added to glucose based WHO ORS and used in the treatment of adult cholera patients, significantly reduced diarrhoeal stool volume and duration of illness. Although the mechanism of diarrhoea is different in cholera, colonic dysfunction has been described ${ }^{10}$ as a result of lack of luminal SCFAs in cholera. ${ }^{11}$

In Bangladesh, fibre containing vegetables and fruits (for example, green plantain and semi-ripe wood apple) are traditionally used in diarrhoeal diseases; with an expectation of early recovery, however, there is a lack of randomised clinical trials to prove their efficacy. Recently, one clinical trial $^{12}$ in the ICDDR examined the effect of cooked green banana and pectin in the treatment of persistent diarrhoea in children. Both green banana and pectin treatment were shown to be beneficial in terms of reducing the duration and stool output in children with persistent diarrhoea. The antidiarrhoeal effect of soluble fibres/unabsorbed carbohydrates is thought to be mediated through their bacterial fermentation, resulting in production of SCFAs. In the colon, SCFAs facilitate the absorption of sodium chloride and water; ${ }^{13-15}$ they also serve as an important source of energy for the colonocytes, ${ }^{16}$ and stimulate epithelial cell proliferation and exert a trophic effect on the colonic as well as small bowel mucosa. ${ }^{17-19}$ In addition, Benefiber slows colonic transit, ${ }^{20}$ which might enhance absorption of salt and water.

Nutritional therapy is an important component in the management of persistent diarrhoea. An algorithmic approach, advised by the WHO, ${ }^{21}$ is followed at most centres, including the ICDDR. In this approach, the initial diet includes a simple, inexpensive, culturally acceptable, less modified (milk based) diet. If this diet fails, it is followed by a lactose and cows' milk free diet, based on rice powder, egg white, and soy oil. If this is unsuccessful a comminuted chicken diet is given. ${ }^{22}$

In our study, we used comminuted chicken diet because this diet is free of fibres and complex carbohydrates. This was intended to examine the effects of Benefiber supplementation. One limitation of this diet is low calorie content $(60 \mathrm{kcal} / 100 \mathrm{ml})$, because glucose was the only carbohydrate present and the concentration of glucose was restricted given its effect in increasing the osmolality. Further studies may be useful to examine the effect of Benefiber added to first and second line diets in the management of persistent diarrhoea.

In conclusion, the present study shows that Benefiber supplemented comminuted chicken diet enhances recovery of children suffering from persistent diarrhoea, which indicates its therapeutic potential in the management of persistent diarrhoea in children.

\section{ACKNOWLEDGEMENTS}

This study was conducted at the ICDDR: Centre for Health and Population Research, with the support (grant no. 8251-1) of Novartis Nutrition, Bern, Switzerland. ICDDR acknowledges with gratitude the commitment of Novartis Nutrition to the Centre's research efforts.

\section{Authors' affiliations}

N H Alam, S A Sarker, P K Bardhan, International Centre for Diarrhoeal Disease Research, Dhaka, Bangladesh

R Meier, GI Unit, Kantonsspital, Liestal, Switzerland

H Schneider, Novartis Nutrition, Bern, Switzerland

N Gyr, University Hospital, Basel, Switzerland

Funding: Novartis Nutrition supported the study, including the patients' study costs and partial salary of the research staff involved in the study.

Competing interests: HS is an employee of Novartis Nutrition.

\section{REFERENCES}

1 World Health Organisation. Persistent diarrhoea in children in developing countries: memorandum from a WHO meeting. Bull WHO 1988;66:709-17.

2 Labenthal E. Prolonged small intestinal mucosal injury as a primary cause of intractable diarrhoea of infancy. In: Labenthal E, ed. Chronic diarrhoea in children. New York: Raven Press, 1984:5-29.

3 Debongnie JC, Phillips SF. Capacity of the human colon to absorb fluid. Gastroenterology 1978;74:698-703.

4 Roediger WE, Moore A. Effect of short chain fatty acids on sodium absorption in isolated human colon perfused through the vascular bed. Dig Dis Sci 1981;26:100-6.

5 Argenzio RA, Miller N, Von Engelhardt W. Effect of volatile fatty acids on water and ion absorption from the goat colon. Am J Physiol 1975;229:997-1002.

6 Hoverstad T. Studies of short chain fatty acids absorption in man. Scand J Gastroenterol 1986;21:257-60.

7 Alam NH, Meier R, Schneider H, et al. Partially hydrolysed guar gumsupplemented oral rehydration solution in the treatment of acute diarrhoea in children. JPGN 2000;31:503-7.

8 World Health Organisation. Programme for control of diarrhoeal diseases. In:A manual for the treatment of acute diarrhoea for use by the physicians and other senior health workers.WHO/CDD/Ser 80.2 ref 1. Geneva: WHO, 1984:25.

9 Ramakrishna BS, Venkataraman S, Srinivasan P, et al. Amylase-resistant starch plus oral rehydration solution for cholera. N Engl J Med 2000;342:308-13.

10 Speelman P, Butler T, Kabir I, et al. Colonic dysfunction during cholera infection. Gastroenterology 1986;91:1164-70.

11 Ramakrishna BS, Mathan VI. Colonic dysfunction in acute diarrhoea: the role of luminal short chain fatty acids. Gut 1993;34:1215-18.

12 Rabbani GH, Teka T, Zaman B, et al. Clinical studies in persistent diarrhoea: dietary management with green banana or pectin in Bangladeshi children. Gastroenterology 2001;121:554-60.

13 Ruppin H, Bar-Meir S, Soergel KH, et al. Absorption of short chain fatty acids by the colon. Gastroenterology 1980;78:1500-7.

14 Argenzio RA, Miller N, Von Engelhardt W. Effect of volatile fatty acids on water and ion absorption from the goat colon. Am J Physiol 1975;229:997-1002. 
15 Binder HJ, Mehta P. Short chain fatty acids stimulate active sodium and chloride absorption in vitro in the rat distal colon. Gastroenterology 1989;96:989-96.

16 Roediger WE. Utilization of nutrient by isolated epithelial cells of the rat colon. Gastroenterology 1982:83:424-9.

17 Sakata T. Effects of indigestible dietary bulk and short chain fatty acids on the tissue weight and epithelial cell proliferation rate of the digestive tract in rats. J Nutr Sci Vitaminol 1986:32:355-62.

18 Roediger WE, Rae DA. Trophic effect of short chain fatty acids on mucosal handling of ions by the defunctioned colon. Br J Surg 1982;69:23-5.
19 Brown RC, Kelleher J, Losowsky MS. The effect of pectin on the structure and function of rat small intestine. Br J Nutr 1979;42:357-65.

20 Meier $\mathbf{R}$, Beglinger $\mathrm{C}$, Schendier $\mathrm{H}$, et al. Effect of a liquid diet with and without soluble fiber supplementation on intestinal transit and cholecystokinin release in volunteers. J Parenter Enteral Nutr 1993;17:231-5.

21 International Working Group on Persistent Diarrhoea. Evaluation of an algorithm for the treatment of persistent diarrhoea: a multicentre study. Bull WHO 1996;74:479-89.

22 Roy SK, Alam AN, Maiid N, et al. Persistent diarrhoea: a preliminary report on clinical features and dietary therapy in Bangladeshi children. J Trop Pediatr 1989;35:55-9.

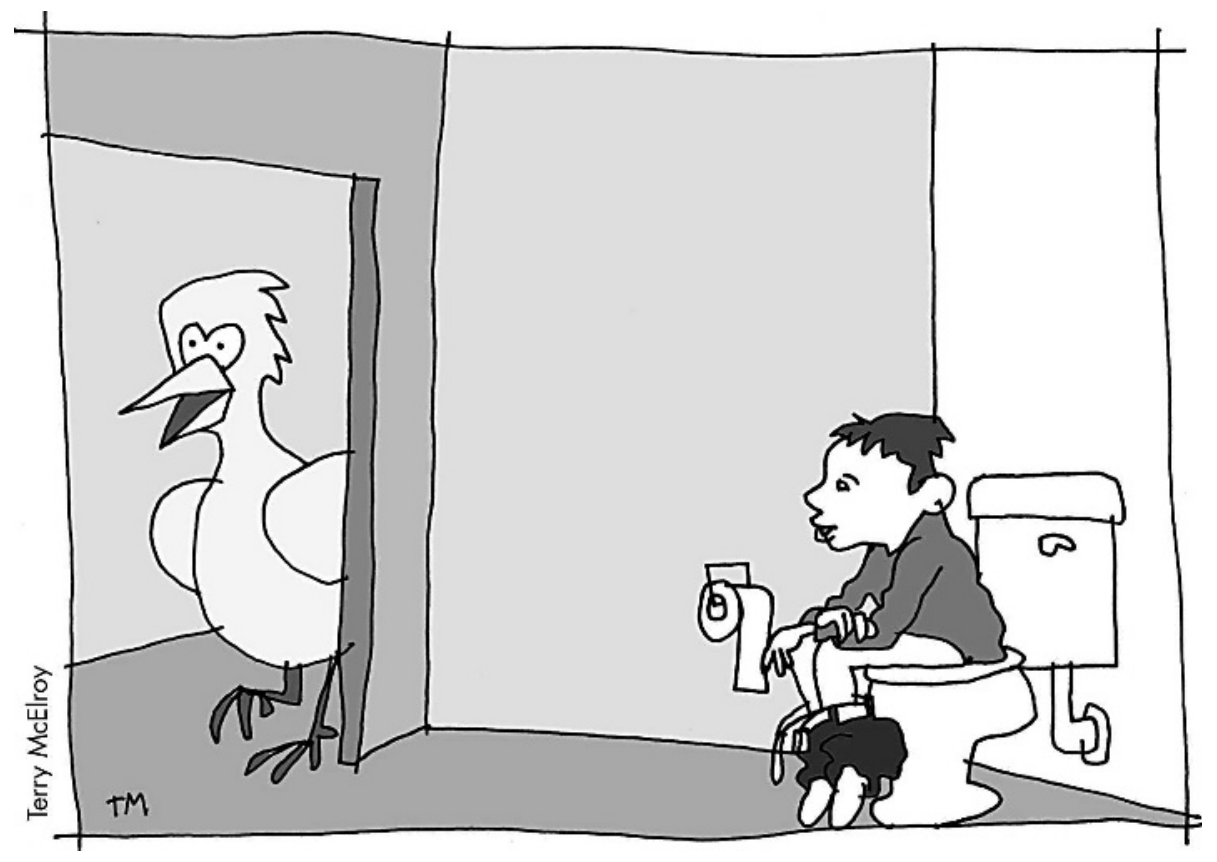

